\title{
Do Primary Care Physicians Lose Contact with Their Colorectal Cancer Patients?
}

\author{
Sharon Dobie, MD, MCP, Barry G. Saver, MD, MPH, Ching-Yun Wang, PhD, \\ Pamela K. Green, PhD, and Laura-Mae Baldwin, MD, MPH
}

Background: Primary care physicians and patients perceive that they lose contact with each other after a cancer diagnosis. The objective of this study was to determine whether colorectal cancer (CRC) patients are less likely to see their primary care physicians after cancer diagnosis.

Methods: This was a longitudinal cohort study using 1993 to 2001 Surveillance Epidemiology and End Results (SEER)-Medicare claims data. Eligible patients were those with stage 0 to 1 and 2 to 3 CRC aged 67 to 89 years at diagnosis. Main measures included the proportion of individuals with a face-toface primary care visit and mean annual primary care visits per patient at baseline and during 5 years after treatment.

Results: Fewer than half of the cancer patients visited with a primary care physician at baseline. In the first year after treatment, patients with stage 0 to $1 \mathrm{CRC}(48.9 \%$ vs $53.3 \% ; P \leq .001)$ and stage 2 to 3 CRC $(43.6 \%$ vs $53.4 \% ; P \leq .001)$ significantly increased their likelihood of visiting a primary care physician from baseline. The proportion of patients with stage 0 to 1 CRC with a primary care visit remained relatively stable, and the proportion of patients with stage 2 to $3 \mathrm{CRC}$ decreased somewhat between the first and fifth year after treatment. The findings for mean annual primary care visits per patient roughly paralleled those for the proportion of individuals with a primary care visit.

Conclusions: Elderly patients with CRC, especially stage 2 to 3 CRC, increase rather than decrease contact with primary care providers after diagnosis. More work is needed to understand the care that different physician specialties provide cancer patients and to support their collaboration. ( $\mathrm{J}$ Am Board Fam Med 2011;24:704-709.)

Keywords: Cancer, Medicare, Primary Health Care, Survivors

Patients diagnosed with cancer enter an intense world of specialized care, including surgery, chemotherapy, and/or radiation therapy, to treat and manage their disease. Although other chronic illness care and preventive health recommendations persist throughout and after cancer treatment, both

This article was externally peer reviewed.

Submitted 24 August 2010; revised 4 June 2011; accepted 15 June 2011.

From the Department of Family Medicine, University of Washington School of Medicine (SD, PKG, L-MB), and the Division of Public Health Sciences, Fred Hutchinson Cancer Research Center (C-YW), Seattle, WA; and the Department of Family Medicine and Community Health, University of Massachusetts Medical School, Worcester, MA (BGS).

Funding: Funding was provided by the National Cancer Institute (grant no. 1 RO1 CA104935).

Conflict of interest: none declared.

Corresponding author: Sharon Dobie, Department of Family Medicine, University of Washington, Box 356390, Seattle, WA 98195-6390 (E-mail: dob@uw.edu). patients and primary care providers perceive that they lose contact with one another after a cancer diagnosis. ${ }^{1-3}$

Patients express the desire to maintain contact with their primary care providers during cancer care. ${ }^{3-5}$ They value these providers' involvement in managing their chronic diseases and acute needs, as well as in providing clarification and support during cancer treatment. ${ }^{1,6}$ At the same time, primary care providers want to stay involved with their patients with cancer, ${ }^{2,5,7-10}$ providing care for treatment side effects, surveillance for recurrence, care of noncancer conditions, and coordination of care. ${ }^{5,7-10}$ Research has demonstrated that management of health screening and chronic illness care after cancer is improved when patients are seen by both cancer specialists and primary care providers, ${ }^{11,12}$ suggesting that an ongoing primary care relationship is important throughout cancer care. 
This longitudinal study examines whether, as patients and providers perceive, patients are less likely to visit their primary care providers after colorectal cancer (CRC) diagnosis relative to before diagnosis. These findings can help to determine whether efforts to ensure that cancer patients receive chronic disease and preventive care should focus on increasing visits to primary care providers or on better coordinating care between cancer care and primary care providers.

\section{Methods}

\section{Data Sources}

This study used Surveillance, Epidemiology, and End Results (SEER) cancer registry data from 1993 to 1999 , linked with 1991 to 2001 Medicare claims. SEER data include patient demographics and cancer type and stage. Medicare data include enrollment dates, health maintenance organization membership, patient demographics, and, for feefor-service beneficiaries, billed claims that include diagnoses, tests, and procedures provided in hospitals, physician offices, and clinics. The American Medical Association Physician Masterfile data linked to the Medicare claims provided physician specialty.

\section{Study Phases}

Study phases included baseline year (12 months before treatment phase); the 7-month treatment phase (month before the diagnosis month through fifth month after diagnosis month); and after treatment (up to five successive 12-month periods after treatment phase). We excluded treatment phase data because we expected justifiably few primary care visits. At the time of this study, standard treatment was surgery alone for stages 0 to 1 CRC patients and surgery plus six cycles of chemotherapy for stage 2 to 3 CRC. We expected the most intensive treatment to be completed for the majority of patients with stage 0 to 3 CRC by the end of the 7-month treatment phase.

\section{Study Population}

Cancer patients were aged 67 to 89 years at diagnosis, had stage 0 to $3 \mathrm{CRC}$ with typical histologies, were not diagnosed at autopsy, and had diagnosis month and year recorded $(\mathrm{n}=64,410)$. We distinguished between patients with early-stage (stages 0 to 1) and later-stage (stages 2 to 3) CRC because prior analysis showed they differed in receipt of noncancer care. ${ }^{13}$

We excluded individuals without continuous enrollment in fee-for-service parts A and B Medicare from baseline through the first year after treatment, leaving 22,161 patients with CRC. Successive years after treatment were included if the individuals remained alive and had continuous enrollment (Table 1). The majority of attrition from both stage 0 to 1 and stage 2 to 3 CRC study groups was because of disenrollment rather than death (more than $80 \%$ for the group with stage 0 to 1 CRC and more than one third for the group with stage 2 to 3 CRC).

\section{Study Variables}

Our study outcomes were proportion of individuals with a primary care physician visit and the mean annual primary care visits per patient during each observation year. Unique visits were claims for face-to-face, nonprocedural services occurring in physician offices, patient homes, or clinics with the same date of service; provider identifier; and beneficiary identifier. Primary care physicians had a primary specialty of family medicine, general internal medicine, or pediatrics (to ensure all physicians with combined general internal medicine/pediatrics training were included), and they had no more specialized, secondary specialty in the American Medical Association Physician Masterfile (or a Centers for Medicare and Medicaid Services specialty code for these specialties from the carrier claims if Masterfile data were unavailable).

For descriptive purposes, we defined demographic variables (patient sex, race); ecological vari-

Table 1. Number of Persons in Each Study Observation Year by Study Group

\begin{tabular}{lcr}
\hline & \multicolumn{2}{c}{ Patients with CRC (n) } \\
\cline { 2 - 3 } & Stage 0-1 & Stage 2-3 \\
\hline Baseline year & 8,865 & 13,296 \\
Treatment phase & 8,865 & 13,296 \\
Years after treatment & & \\
1 & 8,865 & 13,296 \\
2 & 7,856 & 11,471 \\
3 & 5,923 & 8,375 \\
4 & 4,384 & 5,943 \\
5 & 3,086 & 4,019 \\
\hline
\end{tabular}

CRC, colorectal cancer. 
ables (ZIP code-based median annual household income); reason for initial Medicare entitlement; and calculated comorbidity using the National Cancer Institute combined CRC-specific comorbidity index ${ }^{14}$ during the year before baseline. This index, an adaptation of Charlson et al's ${ }^{15}$ comorbidity index, is based on 13 conditions identified from diagnoses on both inpatient and outpatient claims (renal disease, congestive heart failure, dementia, chronic pulmonary disease, cerebrovascular disease, paralysis, diabetes with and without complications, peripheral vascular disease, rheumatologic disease, acute myocardial infarction, prior myocardial infarction, ulcer disease) and uses weights specific to patients with CRC.

\section{Analysis}

We described the cancer patient groups (patients with stage 0 to $1 \mathrm{CRC}$ and patient with stage 2 to 3 CRC) then calculated the proportion of individuals with primary care visits and the mean annual primary care visits per patient by group during the baseline year and for each observation year after the treatment phase. We analyzed whether there were differences over time within each study group using $\chi^{2}$ and standard $t$ tests, as appropriate.

\section{Results}

The majority of both stage 0 to 1 CRC and stage 2 to 3 CRC study groups were women, white, eligible for Medicare because of age, and living in ZIP codes with more than $\$ 30,000$ in median household income (Table 2).

Fewer than half of the two study groups visited with a primary care physician during the baseline year (Figure 1). During the first year after treatment, patients with stage 0 to $1(48.9 \%$ vs $53.3 \%$; $P \leq .001)$ and stage 2 to $3(43.6 \%$ vs $53.4 \% ; P \leq$ .001) CRC significantly increased their likelihood of visiting a primary care physician from baseline. Patients with stage 0 to 1 CRC had a minor decrease in the second year after treatment $(53.3 \%$ to $51.3 \% ; P \leq .01)$ then remained stable until the fifth year after treatment. The proportion of patients with stage 2 to 3 CRC who visited a primary care physician decreased between the first and fourth year after treatment $(53.4 \%$ vs $49.6 \% ; P \leq .001)$ then remained stable in the fifth year after treatment $(50.8 \%)$. These lower proportions of stage 2
Table 2. Characteristics of the Study Population at Baseline

\begin{tabular}{|c|c|c|}
\hline \multirow[b]{2}{*}{ Characteristics } & \multicolumn{2}{|c|}{ Patients with CRC (\%) } \\
\hline & $\begin{array}{l}\text { Stage } 0-1 \\
(\mathrm{n}=8865)\end{array}$ & $\begin{array}{c}\text { Stage } 2-3 \\
(\mathrm{n}=13,296)\end{array}$ \\
\hline \multicolumn{3}{|l|}{ Age (years) } \\
\hline $65-69$ & 21.4 & 19.0 \\
\hline $70-74$ & 29.0 & 28.2 \\
\hline $75-79$ & 27.1 & 26.6 \\
\hline $80-89$ & 22.5 & 26.2 \\
\hline \multicolumn{3}{|l|}{ Sex } \\
\hline Female & 53.9 & 56.6 \\
\hline \multicolumn{3}{|l|}{ Race/ethnicity } \\
\hline White & 86.6 & 87.2 \\
\hline African American & 6.3 & 6.1 \\
\hline Hispanic & 1.0 & 1.1 \\
\hline Asian & 3.5 & 3.3 \\
\hline Other & 2.6 & 2.3 \\
\hline \multicolumn{3}{|c|}{ NCI comorbidity index score } \\
\hline$<0.0$ & 76.8 & 79.0 \\
\hline $0.0-0.5$ & 13.2 & 12.0 \\
\hline $0.5-1.0$ & 6.2 & 5.8 \\
\hline$>1.0$ & 3.8 & 3.1 \\
\hline \multicolumn{3}{|c|}{ Reason for Medicare eligibility } \\
\hline Renal disease/disability & 5.9 & 5.3 \\
\hline Age $\geq 65$ years & 94.1 & 94.7 \\
\hline \multicolumn{3}{|c|}{$\begin{array}{l}\text { Median household income in } \\
\text { residence ZIP code* }\end{array}$} \\
\hline$\leq \$ 30,000$ & 8.2 & 8.6 \\
\hline$\$ 30,001-\$ 45,000$ & 38.4 & 39.0 \\
\hline$>\$ 45,000$ & 53.4 & 52.4 \\
\hline
\end{tabular}

*Median household income in residence ZIP code statistics have missing values: stage $0-1,210$; stage $2-3,301$.

NCI, National Cancer Institute; CRC, colorectal cancer.

to 3 CRC visiting a primary care physician in the fourth and fifth years after treatment were still significantly higher than the proportion at baseline. The findings for the outcome-mean annual primary care visits per patient-paralleled the findings for proportion of individuals with a primary care visit (Figure 2).

\section{Discussion}

Our study asked, Do patients continue to visit primary care physicians after a colorectal cancer diagnosis? Contrary to patients' and primary care providers' perceptions, ${ }^{1-3}$ this study found that older patients with CRC increase their likelihood of seeing primary care physicians after cancer diagnosis and treatment, and this higher rate continues 
Figure 1. Proportion of colorectal cancer patients with a primary care visit over the observation period. // indicates that the beginning of observation year 1 lags by 7 months from the end of the baseline year. We excluded this treatment phase because we expected primary care visits to be superseded by frequent cancer-related care during this time.

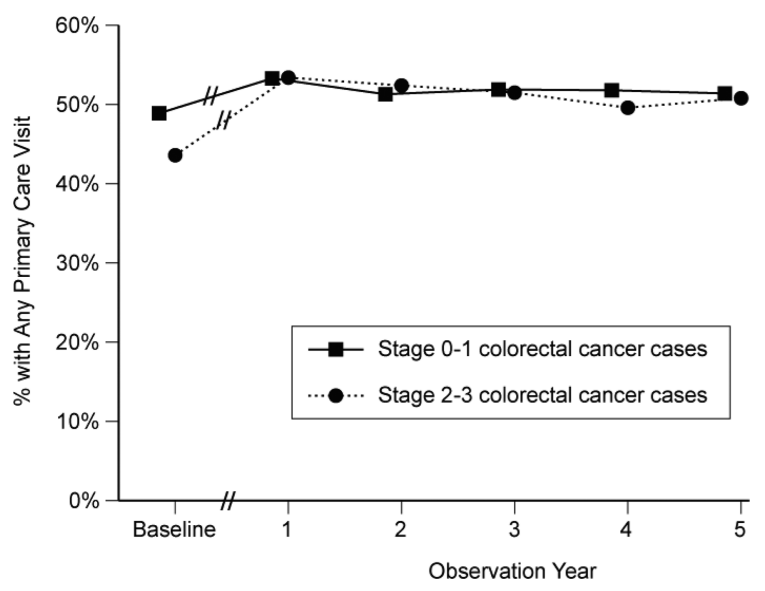

through the first 5 years after diagnosis and treatment. Other research has found that, over time, cancer survivors have stable rates of seeing primary care providers, ${ }^{12,16}$ but this study is the first to demonstrate the increase in primary care use after cancer diagnosis.

Why do patients and primary care physicians perceive a loss of contact with one another when their contact actually increases initially, especially for CRC patients with more advanced disease? One hypothesis is that, for patients with CRC, visits with primary care physicians are relatively infrequent compared with visits with cancer care specialists. In turn, primary care physicians may perceive a loss of contact if they know their patients are in intensive treatment yet they receive limited or delayed information about that care. ${ }^{7,8,10}$ Lastly, we did not measure visits during the 7-month treatment phase because we expected primary care visits to be superseded by frequent cancer-related care during this time. Perhaps a lower primary care visit rate during that period fuels provider perception that they lose contact with their patients.

Despite their more intensive cancer treatment regimens, patients with stage 2 to $3 \mathrm{CRC}$ are significantly more likely to see primary care providers after cancer diagnosis, suggesting that diagnosis has altered their care-seeking behavior. Perhaps, as
Denmark-Wahnefried et a ${ }^{17}$ suggest, cancer diagnosis provides a teachable moment when individuals are more willing to hear health promotion messages and participate in activities and lifestyle modifications that optimize health. Primary care visits may represent important opportunities to influence prevention and management of other health conditions as well as monitor for cancer recurrence and short- and long-term treatment effects.

This study's limitations include lack of generalizability because of inclusion of only elderly patients with CRC who receive fee-for-service Medicare. Younger cancer patients with fewer chronic conditions may visit primary care physicians differently. Other studies have shown greater proportions of patients have access to or see primary care physicians; they include more specialties and a broader range of visit codes. ${ }^{16,18}$ Our study, using only face-to-face nonprocedural visits, may have underestimated the total primary care visits for both CRC patients and controls.

Attrition in the stage 0 to 1 and stage 2 to 3 CRC groups over the study years was dominated by disenrollment from Medicare fee for service to Medicare health maintenance organizations. Research has demonstrated that patients moving from enrollment in Medicare fee for service into health maintenance organizations are health-

Figure 2. Mean annual primary care visits per patient among colorectal cancer patients over the observation period. // indicates that the beginning of observation year 1 lags by 7 months from the end of the baseline year. We excluded this treatment phase because we expected primary care visits to be superseded by frequent cancer-related care during this time.

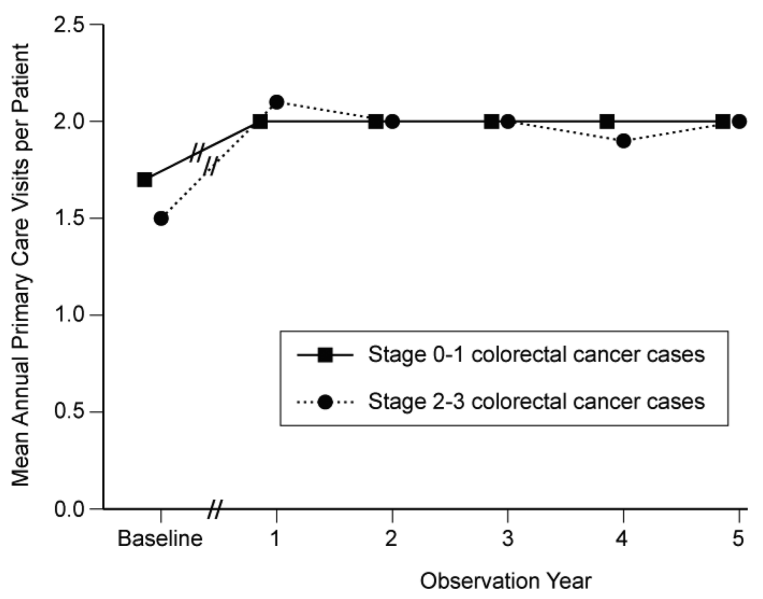


ier. ${ }^{19,20}$ Thus, because of this phenomenon and aging, over time the comorbidity of our study cohorts and their visits to primary care physicians will increase. However, between baseline and the first observation year, when the study sample had no shifts from death and disenrollment, there was a significant increase in primary care visits. Regardless of these influences, our study's conclusion does not change: primary care physicians continue to see their patients with CRC as much or more after diagnosis.

Patients with CRC receive ongoing primary care after cancer treatment; for patients with stage 2 to $3 \mathrm{CRC}$, it is more often than before the diagnosis. Some work suggests that patients seeing both their oncology team and their primary care physicians obtain the greatest benefit in delivery of preventive services. ${ }^{12}$ Other work describes an independent benefit of care provided by primary care providers. ${ }^{21-24}$ Future research is needed to characterize the components of care provided by different specialties to cancer patients and to seek ways to support the collaboration between the physicians involved in their care.

The authors would like to thank Barbara Matthews for her assistance in developing the database used in this research; Denise Lishner for carefully documenting the work of the research team; and Roger A. Rosenblatt, MD, MPH, MFR, for his advice on study development and design.

\section{References}

1. Sisler JJ, Brown JB, Stewart M. Family physicians' roles in cancer care. Survey of patients on a provincial cancer registry. Can Fam Physician 2004;50: 889-96.

2. Anvik T, Holtedahl KA, Mikalsen H. "When patients have cancer, they stop seeing me": the role of the general practitioner in early follow-up of patients with cancer-a qualitative study. BMC Fam Pract 2006;7:19.

3. Miedema B, MacDonald I, Tatemichi S. Cancer follow-up care. Patients' perspectives. Can Fam Physician 2003;49:890-5.

4. Grunfeld E, Fitzpatrick R, Mant D, et al. Comparison of breast cancer patient satisfaction with follow-up in primary care versus specialist care: results from a randomized controlled trial. Br J Gen Pract 1999;49:705-10.

5. Kendall M, Boyd K, Campbell C, et al. How do people with cancer wish to be cared for in primary care? Serial discussion groups of patients and carers. Fam Pract 2006;23:644-50.
6. Bulsara C, Ward AM, Joske D. Patient perceptions of the GP role in cancer management. Aust Fam Physician 2005;34:299-300, 302.

7. Del Giudice ME, Grunfeld E, Harvey BJ, Piliotis E, Verma S. Primary care physicians' views of routine follow-up care of cancer survivors. J Clin Oncol 2009;27:3338-45.

8. Dworkind M, Towers A, Murnaghan D, Guibert R, Iverson D. Communication between family physicians and oncologists: qualitative results of an exploratory study. Cancer Prev Control 1999;3:137-44.

9. Zitzelsberger L, Grunfeld E, Graham ID. Family physicians' perspectives on practice guidelines related to cancer control. BMC Fam Pract 2004;5:25.

10. Nissen MJ, Beran MS, Lee MW, Mehta SR, Pine DA, Swenson KK. Views of primary care providers on follow-up care of cancer patients. Fam Med 2007; 39:477-82.

11. Oeffinger KC, McCabe MS. Models for delivering survivorship care. J Clin Oncol 2006;24:5117-24.

12. Earle CC, Neville BA. Under use of necessary care among cancer survivors. Cancer 2004;101:1712-9.

13. Baldwin LM, Dobie SA, Cai Y, Saver BG, Green PK, Wang CY. Receipt of general medical care by colorectal cancer patients: a longitudinal study. J Am Board Fam Med 2011;24:57-68.

14. Klabunde CN, Potosky AL, Legler JM, Warren JL. Development of a comorbidity index using physician claims data. J Clin Epidemiol 2000;53: $1258-67$.

15. Charlson ME, Pompei P, Ales KL, MacKenzie CR. A new method of classifying prognostic comorbidity in longitudinal studies: development and validation. J Chronic Dis 1987;40:373-83.

16. Snyder CF, Earle CC, Herbert RJ, Neville BA, Blackford AL, Frick KD. Preventive care for colorectal cancer survivors: a 5-year longitudinal study. J Clin Oncol 2008;26:1073-9.

17. Demark-Wahnefried W, Aziz NM, Rowland JH, Pinto BM. Riding the crest of the teachable moment: promoting long-term health after the diagnosis of cancer. J Clin Oncol 2005;23:5814-30.

18. Okoro CA, Strine TW, Young SL, Balluz LS, Mokdad AH. Access to health care among older adults and receipt of preventive services. Results from the Behavioral Risk Factor Surveillance System, 2002. Prev Med 2005;40:337-43.

19. U.S. Government Accountability Office. Medicare Advantage: characteristics, financial risks and disenrollment rates of beneficiaries in private fee-for-service plans. Washington, DC: U.S. Government Accountability Office; 2008.

20. Maciejewski ML, Dowd B, Call KT, Feldman R. Comparing mortality and time until death for medicare HMO and FFS beneficiaries. Health Serv Res 2001;35:1245-65.

21. Holtedahl K, Norum J, Anvik T, Richardsen E. Do cancer patients benefit from short-term contact with 
a general practitioner following cancer treatment? A randomised, controlled study. Support Care Cancer 2005;13:949-56.

22. Grunfeld E, Levine MN, Julian JA, et al. Randomized trial of long-term follow-up for early-stage breast cancer: a comparison of family physician versus specialist care. J Clin Oncol 2006;24:848-55.
23. Grunfeld E, Mant D, Yudkin P, et al. Routine follow up of breast cancer in primary care: randomised trial. BMJ 1996;313:665-9.

24. Burge F, Lawson B, Johnston G. Family physician continuity of care and emergency department use in end-of-life cancer care. Med Care 2003;41:9921001 . 